\title{
Current Role of Multi-detector Computed Tomography (MDCT) in Diagnosis of Pulmonary Embolism
}

\author{
Kishan Singh Rawat • T. B. S. Buxi • \\ Hariprasad Sudarsan • Anurag Yadav • \\ Samarjit Singh Ghuman
}

Published online: 6 September 2014

(C) Springer Science+Business Media New York 2014

\begin{abstract}
Pulmonary embolism (PE) is a common and serious condition, and if not diagnosed timely may lead to the death of the patient. Imaging plays a major role in the diagnosis of the PE. Since the past decade multi-detector computed tomographic pulmonary angiography is playing a major role in the diagnosis of PE due to its high accuracy. PE may be acute or chronic. PE may cause complete or partial obstruction of the pulmonary arteries. In acute PE, complete obstruction of the segmental and subsegmental branches of pulmonary artery causes enlargement of the vessel. Completely obstructed segmental and subsegmental vessels are smaller in size in the chronic PE. Chronic PE may also lead to the poststenotic dilatation of the obstructed vessel and development of the systemic collateral vessels. Multi-detector computed tomographic pulmonary angiography may be used to assess the clot resolution and severity of the PE.
\end{abstract}

This article is part of topical collection on CT Pulmonary Embolus and Diffuse Lung Disease.

\author{
K. S. Rawat $(\bowtie) \cdot$ T. B. S. Buxi · H. Sudarsan · A. Yadav · \\ S. S. Ghuman \\ Sir Ganga Ram Hospital, Rajinder Nagar, New Delhi 110060, \\ India \\ e-mail: ksrawat14@rediffmail.com \\ T. B. S. Buxi \\ e-mail: tbsbuxi@yahoo.com \\ H. Sudarsan \\ e-mail: s_hariprasad@live.com \\ A. Yadav \\ e-mail: anu_nalin@hotmail.com \\ S. S. Ghuman \\ e-mail: samarjitghuman@gmail.com
}

Keywords Pulmonary embolism - Multi-detector computed tomographic pulmonary angiography . Pulmonary artery $\cdot$ Poststenotic dilatation - Systemic collateral vessels

\section{Introduction}

Pulmonary embolism (PE) is the third most common cause of acute cardiovascular death, after myocardial infarction and stroke $[1,2]$. PE is one of the common complications in the hospitals and contributes to $5-10 \%$ of deaths in hospitals [3-5]. Risk factors for PE are prolonged immobility, surgery, pregnancy, trauma, estrogen therapy, malignancy, congestive heart failure, and acquired or congenital defects in blood coagulation factors and age. PE may be acute or chronic with partial or complete intraluminal filling defects. Timely and accurate diagnosis of PE is crucial to improve the patient outcome and to reduce the mortality. The widespread availability of multi-detector computed tomography (MDCT) and rapid advances in its technology over the last decade has made the MDCT pulmonary angiography the primary imaging modality because of its high diagnostic accuracy and short scanning time. In this article, we review the role of MDCT in the diagnosis of acute and chronic PE, and assessment of the clot resolution and severity of PE.

\section{MDCT in the Diagnosis of Pulmonary Embolism}

The two frontline imaging modalities for the diagnosis of $\mathrm{PE}$ are ventilation-perfusion (V/Q) scintigraphy and MDCT pulmonary angiography. V/Q scintigraphy has high sensitivity but very low specificity [6]. CT pulmonary angiography 
has now replaced the V/Q scintigraphy as the primary imaging modality in the diagnosis of suspected PE [7]. MDCT pulmonary angiography allows not only the comprehensive assessment of PE but also the other causes of acute chest pain and any underlying lung disease.

MDCT with its improved spatial and temporal resolution has increased the sensitivity and specificity in the diagnosis of PE. Increased use of MDCT in PE confirms that CT has now been accepted as primary imaging investigation in the suspected PE in many institutions [8]. In the early 1990 s, RemyJardin et al. [9] reported the sensitivity and specificity of CT as 90 and $96 \%$, respectively, for the diagnosis of central and lobar pulmonary thromboembolism, but did not describe the segmental and subsegmental pulmonary thromboembolism. In 1995, Goodman et al. compared helical CT angiography with pulmonary angiography in the patients with suspected pulmonary thromboembolism and evaluated the segmental and subsegmental pulmonary thromboembolism. They reported the sensitivity of $86 \%$, and specificity of $92 \%$, for CT angiography in the detection of pulmonary thromboembolism in the central and segmental pulmonary arteries. When they included the subsegmental branches in their study, they found specificity of $89 \%$, but sensitivity decreased to $63 \%$ [10]. Later, with the introduction of 64-slice MDCT, rate of detection of segmental and subsegmental pulmonary thromboembolism was significantly increased [11, 12]. In 2006, the multicenter PIOPED II study found that the sensitivity of CT angiography was $83 \%$ and the specificity was $96 \%$ in the diagnosis of PE [13].

Newer techniques like computer aided detection (CAD) are being evaluated to aid in the detection of PE. Wittenberg et al. [14•] in a retrospective study of 209 patients reported that $\mathrm{CAD}$ has the potential to increase the sensitivity for the detection of segmental and subsegmental pulmonary emboli without significantly compromising the specificity.

\section{MDCT Technique}

128-slice multi-detector CT scanner (Philips Ingenuity, Philips Medical System, Netherlands) was used to acquire images of thorax during breath hold after inspiration in caudocranial direction. CT parameters used for pulmonary angiography are shown in Table 1 . An 18- or 20-gage cannula was used for intravenous access into an antecubital vein. $70 \mathrm{ml}$ of contrast medium Omnipaque (Iohexol 300, GE Healthcare, Shanghai, China) was administered intravenously with the help of Power injector (Medrad) at a rate of $4 \mathrm{ml} / \mathrm{sec}$. Bolus tracking method was used with a cursor in the main pulmonary artery at a preset threshold of $120 \mathrm{HU}$ to trigger the scanning for optimum enhancement of pulmonary arteries.
Table 1 Protocol for 128-slice CT in pulmonary embolism

\begin{tabular}{ll}
\hline Detector width & $0.625 \mathrm{~mm}$ \\
Slice thickness & $0.90 \mathrm{~mm}$ \\
Slice increment & $0.45 \mathrm{~mm}$ \\
Pitch & 1.29 \\
Rotation time & $0.40 \mathrm{~s}$ \\
Field of view & $336.0 \mathrm{~mm}$ \\
Voltage & $100 \mathrm{kV}$ \\
Current & $299 \mathrm{~mA}$ \\
\hline
\end{tabular}

\section{Interpretation of CT Images}

CT images acquired were viewed on Philips EBW 4.5 workstation in the axial, coronal, and sagittal planes with two different gray scales for lung window (window width/ level- 1,600/-600 HU) and mediastinal window (360/ $60 \mathrm{HU})$ settings. Volumetric data acquisition helps in using multiplanar reformatted images in the multiple planes including sagittal, coronal, curved planer, and paddle wheel view and increases the sensitivity for the detection of PE. Paddle wheel view is a reconstruction technique, in which multiple planar slabs are obtained which pivot around a central horizontal axis between the lung hila [15] (Fig. 1). In 2003, Chiang et al. [16] in a series of five patients of PE reported that the paddle wheel view had a significantly higher percentage of detection of PE in comparison to the coronal reformations that were obtained with same slab thickness.

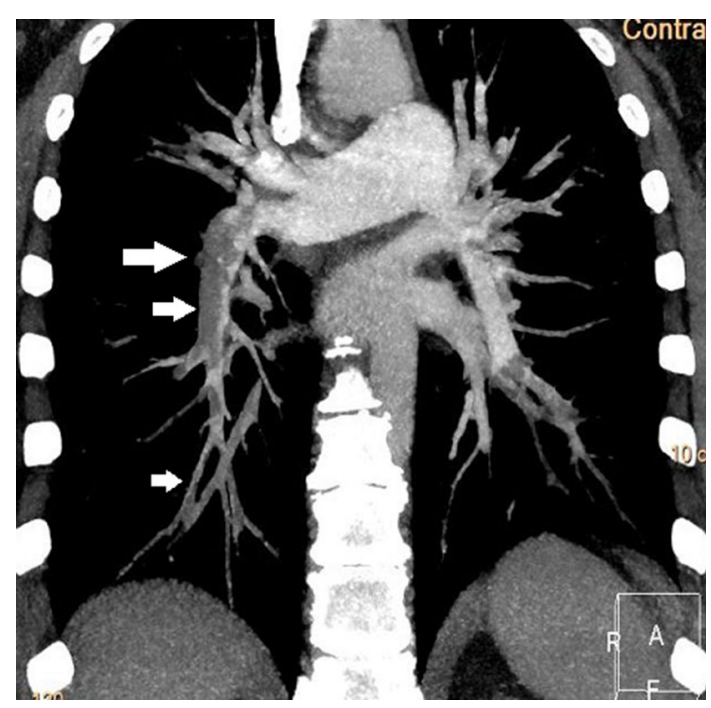

Fig. 1 MDCT pulmonary angiography image using paddlewheel reformation shows the full extent of pulmonary embolism (arrows) in right lower lobe pulmonary artery extending into the segmental and subsegmental branches. Paddlewheel view demonstrates the entire extent of PE in the vessel in continuity from the hilum into the distal subsegmental branches 

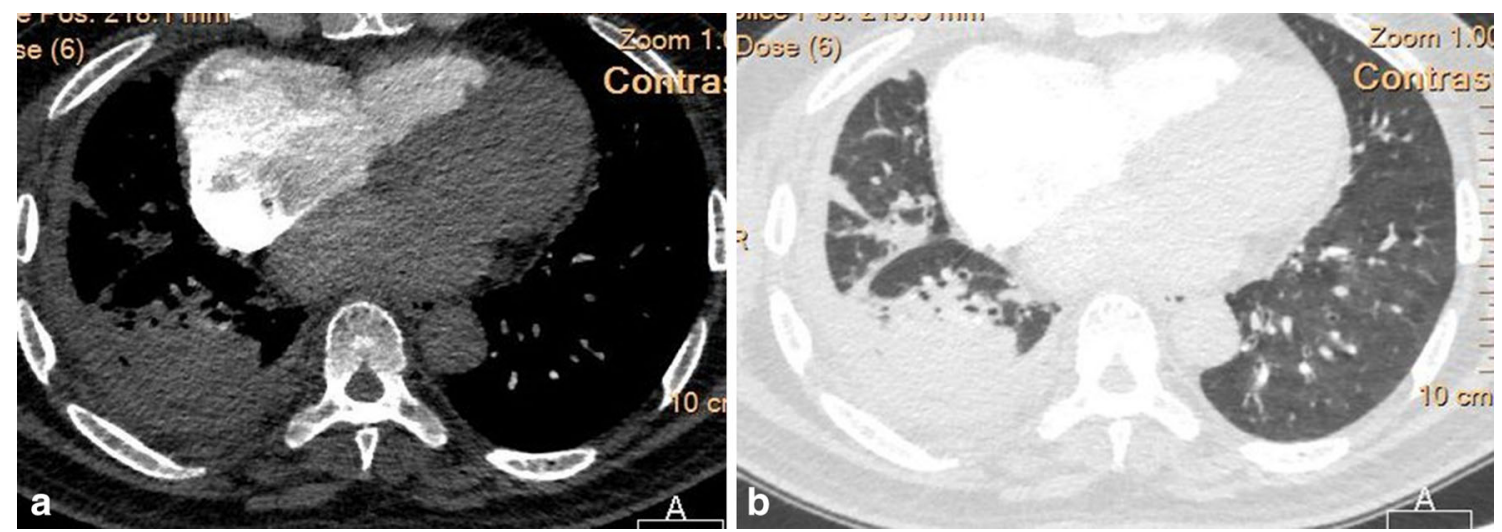

Fig. 2 A 45-year-old male who presented with cough and breathlessness. MDCT images in axial plane in mediastinal (a) and lung window (b) settings showing empyema on the right side with consolidation collapse in the underlying lung parenchyma

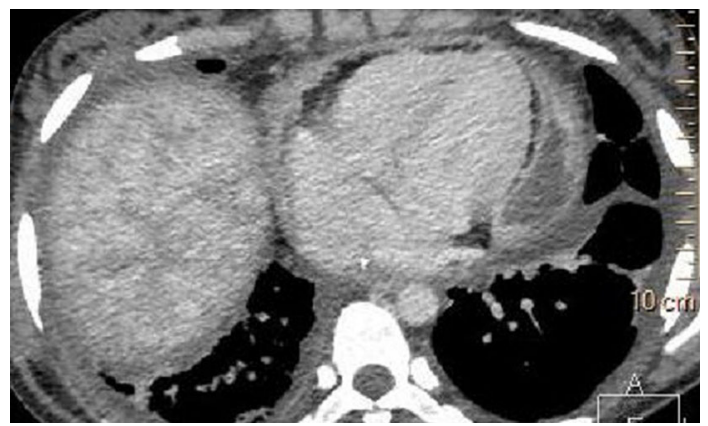

Fig. 3 A 53-year-old female presented with chest pain and breathlessness. CT image in mediastinal window shows pericardial effusion with pericardial thickening

On CT pulmonary angiography, main pulmonary artery, right and left pulmonary arteries, and their lobar, segmental, and subsegmental branches are evaluated for the pulmonary emboli which are seen as non-enhancing low-

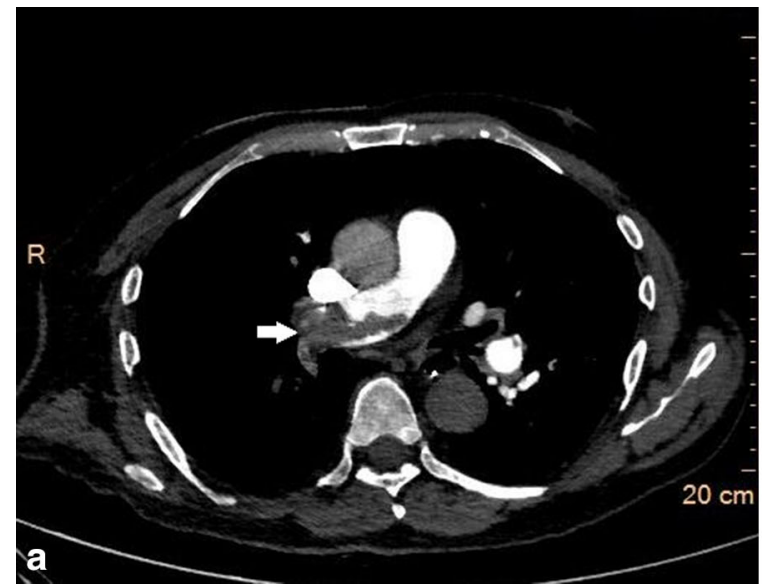

Fig. 4 MDCT pulmonary angiography of a 60-year-old male presenting with acute onset of chest pain and dyspnea. a MDCT axial image showing complete obstruction of the right pulmonary attenuation filling defects. PE may lead to the lung parenchymal changes due to vascular occlusion. Hence, lung parenchymal evaluation in the lung window setting is essential. Lung parenchyma as well as mediastinum evaluation is also necessary because more than $70 \%$ patients, suspected of PE, do not show pulmonary emboli in the CT pulmonary angiography, and alternate diagnoses of diseases of lung parenchyma, pleura (Fig. 2), pericardium (Fig. 3), and mediastinum are possible in such patients [17].

\section{Acute Pulmonary Embolism}

Direct Signs

Acute PE leads to either complete or partial arterial occlusion. In complete arterial occlusion, the vessel does not show contrast because of non-enhancing low-attenuation filling

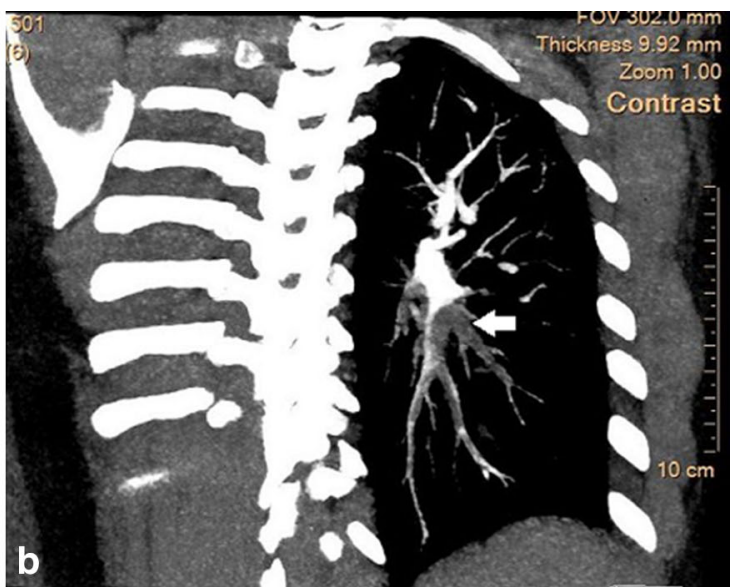

artery by the embolus. b MDCT axial image showing complete obstruction of a segmental artery of left lower lobe 

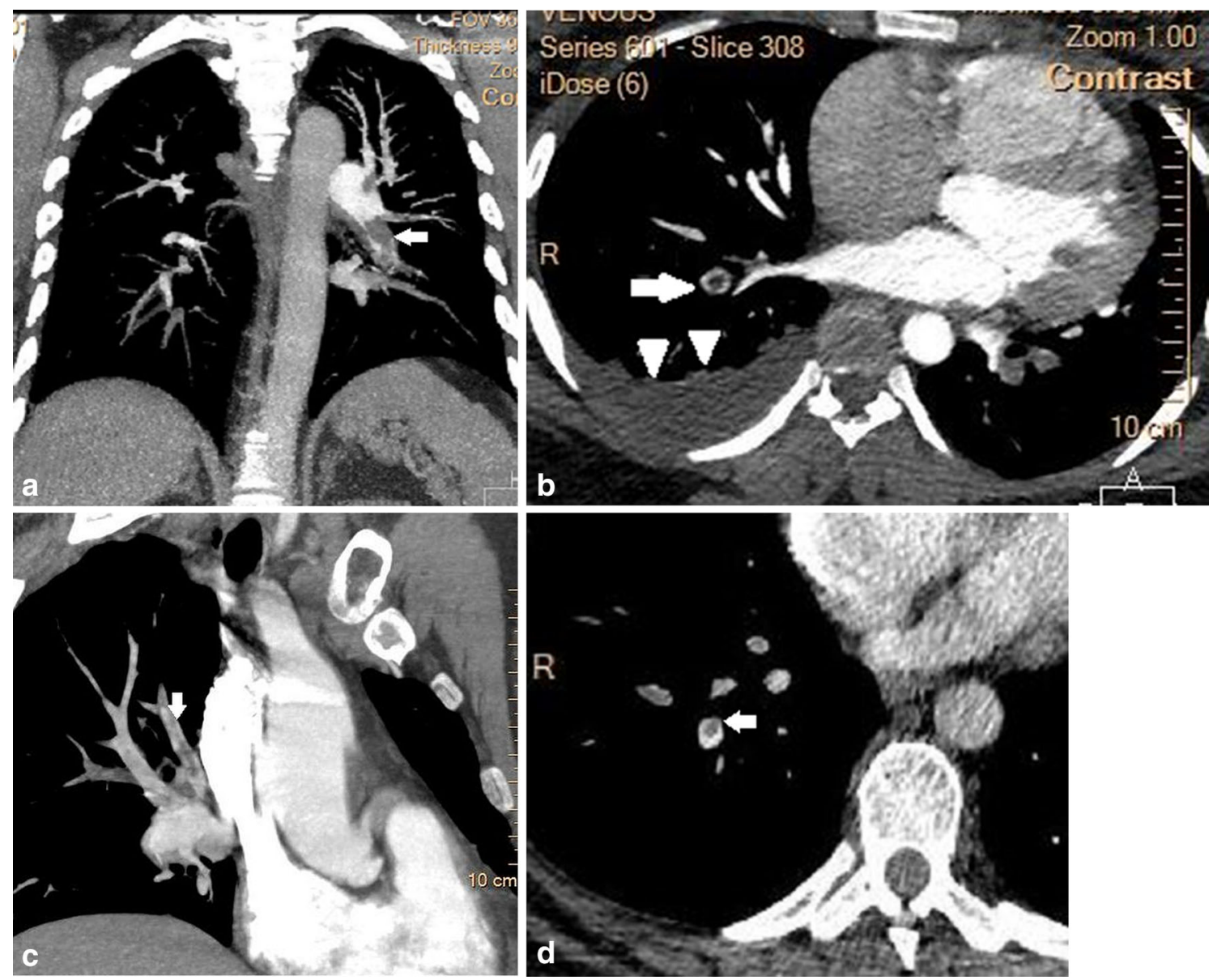

Fig. 5 a CT pulmonary angiography image of a 65-year-old man presented with chest pain showing a partial thrombus in the lumen of the left lower lobe pulmonary artery. b CT pulmonary angiography image of a 33-year-old male who presented with acute onset breathlessness and chest pain shows a central thrombus in the segmental artery of right lower lobe, and making a "polo mint sign" (arrow) and pleural effusion on the right side (arrow heads). c CT

defect. The completely occluded artery shows concave filling defect within the column of contrast material with trailing edge of the embolus, and the vessel may be enlarged distal to the point of complete occlusion as compared to the adjacent normal vessel (Fig. 4). In partial obstruction, the embolus may be central or eccentric (Fig. 5). The central partial filling defect is surrounded by intraluminal contrast material, and it may be seen as "polo mint" sign on axial image and as "railroad track" sign on longitudinal image (Fig. 5). The partial filling defect makes acute angles with the vessel wall (Fig. 5).

\section{Indirect Signs}

Wedge-shaped consolidation in the subpleural region of lung with its broad base along the pleural surface and a image of a patient who presented with breathlessness showing an acute pulmonary embolus that causes a partial filling defect surrounded by contrast material and producing "railway track sign" (arrow). d CT image in a patient of acute pulmonary embolism, presented with chest pain, shows an eccentrically located thrombus that forms acute angles with the vessel wall (arrow)

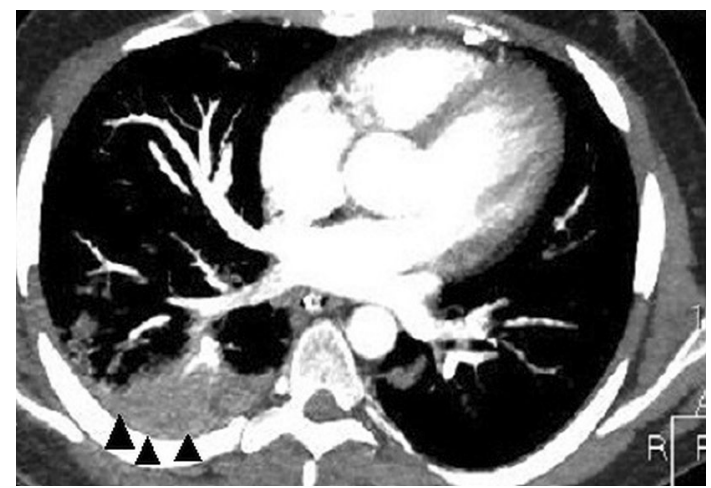

Fig. 6 CT angiography image in a 35-year-old male patient of acute pulmonary embolism who presented with breathlessness. CT image showing a wedge-shaped pleural-based opacity in the right basal segments (arrow heads) 

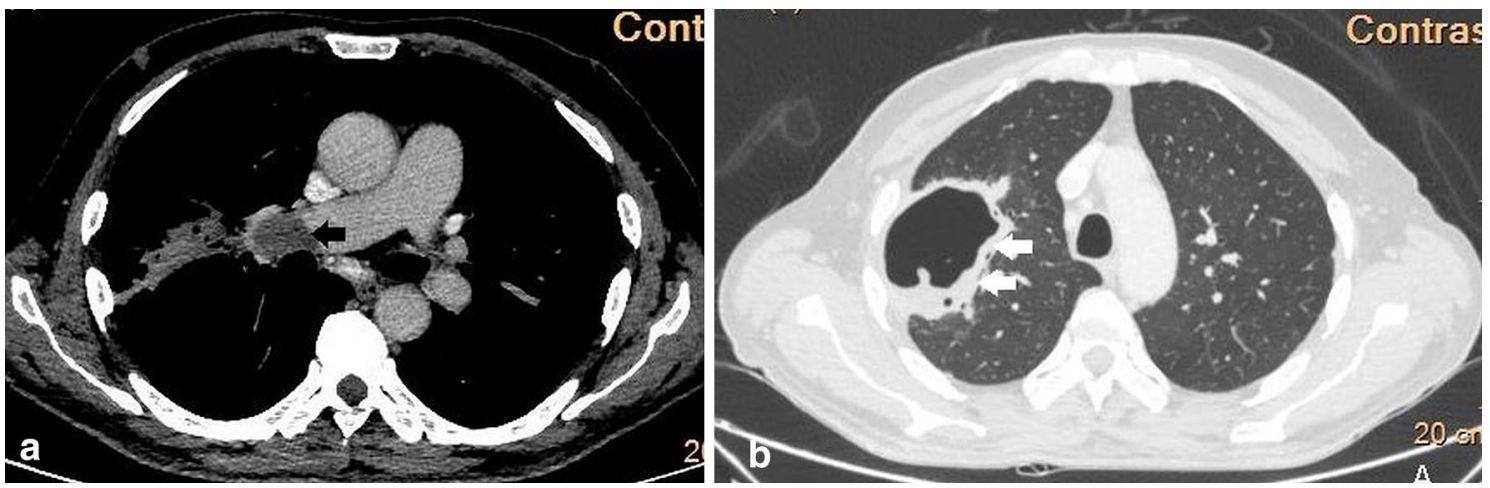

Fig. 7 a A 45-year-old female presented with chest pain and dyspnoea. a CT image in mediastinal window shows thrombus in the right pulmonary artery. b CT image in lung window shows cavitary lesion (arrows)

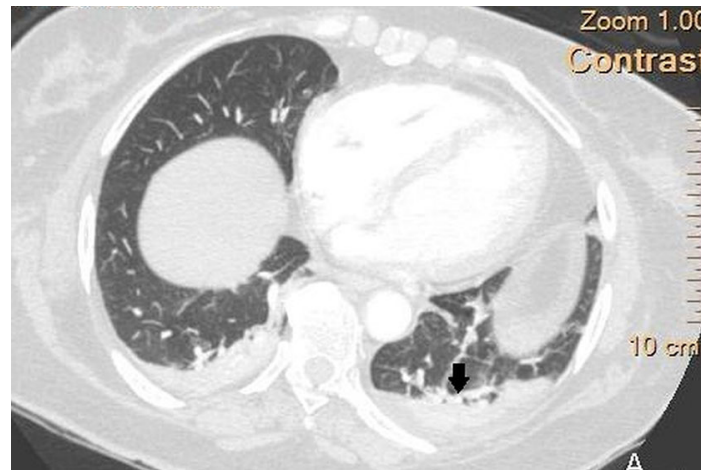

Fig. 8 A 55-year-old male patient of acute pulmonary embolism who presented with chest pain, CT image in lung window, shows consolidation with air lucencies (arrow) in the posterobasal segment of left lower lobe

rounded central margin known as Hampton' hump is a well-recognized indirect sign, seen in acute $\mathrm{PE}$ which may represent infarct [18] (Fig. 6). Oligaemia of lung parenchyma distal to the occluded artery, known as Westermark sign, can be seen in acute PE [19]. Acute PE can also lead to the mosaic attenuation areas in the lung fields on CT due to nonuniform arterial perfusion of lung parenchyma, though this sign is more common in the chronic PE [20]. Other indirect signs of acute PE are peripheral consolidation without air bronchogram [21], the melting sign of resolving infarct [22], cavitary lesion (Fig. 7) [23], and consolidation containing air lucencies [24] (Fig. 8).

\section{Chronic Pulmonary Embolism}

Pulmonary emboli do not resolve completely in a small number of patients, and they persist as endotheliazed fibrotic stenosis of pulmonary arteries and lead to chronic $\mathrm{PE}$, and manifest as pulmonary hypertension and cor pulmonale [25]. Reduced blood flow in pulmonary arteries

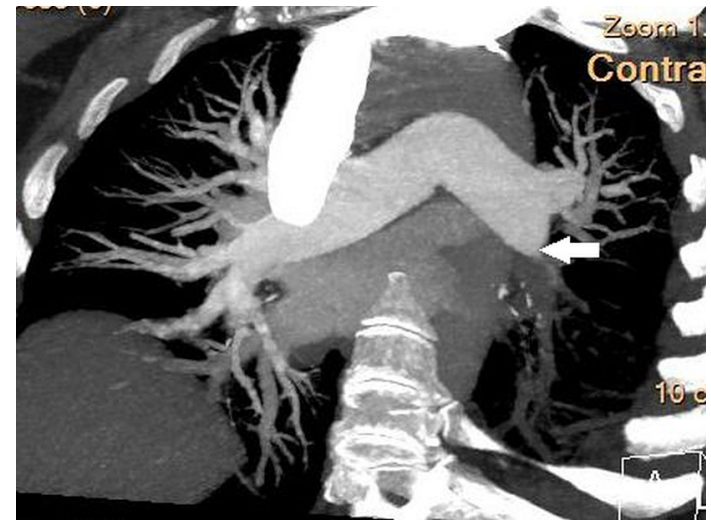

Fig. 9 Coronal reformatted image of a 62-year-old male with chronic pulmonary thromboembolism showing pouch defect (arrow)

may lead to hypertrophy of bronchial arteries and development of systemic collateral vessels. The incidence of chronic PE following acute PE is 1.3 to $3.8 \%$ [26].

$\mathrm{CT}$ findings in chronic PE are described as vascular and lung parenchymal.

\section{Vascular CT Findings}

Direct Vascular Signs

\section{Complete Pulmonary Artery Obstruction}

Chronic PE may lead to complete cutoff of vessel with contrast-filled vessel which has a convex margin of contrast material, and this feature has been described as "pouch defect" on pulmonary CT angiography [27] (Fig. 9). Vessel distal to the obstruction is commonly smaller in diameter and has irregular outline due to the contraction of chronic embolus [28]. 


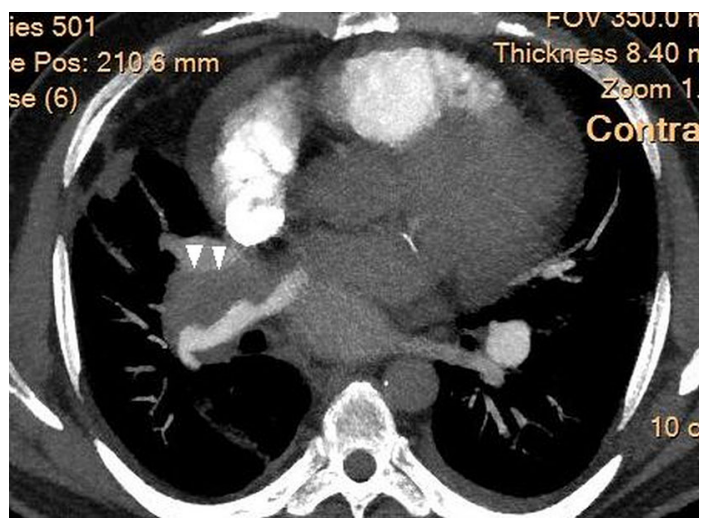

Fig. 10 CT angiography of a 75-year-old male with chronic pulmonary thromboembolism showing partial obstruction in the right lower lobe pulmonary artery with intimal irregularity

\section{Partial Pulmonary Artery Obstruction}

Chronic-organized embolus may cause partial obstruction, intimal irregularity, band, web, and abrupt vessel narrowing. The organized embolus with partial obstruction appears as vessel wall thickening and may cause intimal irregularity [29] (Fig. 10). A band is a linear filling defect attached to the vessel wall at both ends and has a free midportion. It is usually less than $0.3 \mathrm{~cm}$ in width and up to $2 \mathrm{~cm}$ in length. It is oriented in the direction of blood flow and along the long axis of the vessel. A web is a network of multiple bands. Bands and webs are seen as linear filling defects surrounded by contrast material on CT pulmonary angiography [30] (Fig. 11). Calcifications may be seen in chronic embolus (Fig. 12).

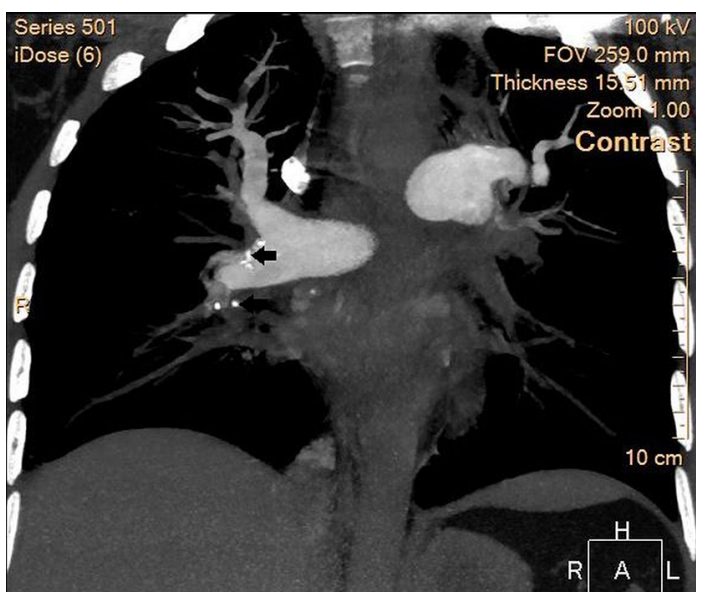

Fig. 12 CT pulmonary angiography image of a 70-year-old female with chronic pulmonary thromboembolism showing calcifications in the right pulmonary artery

Indirect Vascular Signs

\section{Tortuous Pulmonary Arteries}

Tortuous pulmonary vessels may be seen in the patients with chronic PE [31] (Fig. 13).

\section{Poststenotic Dilatation of Pulmonary Artery}

On CT pulmonary angiography, poststenotic dilatation or aneurysm of pulmonary vessels is commonly seen in chronic thromboembolic disease [32] (Fig. 13).

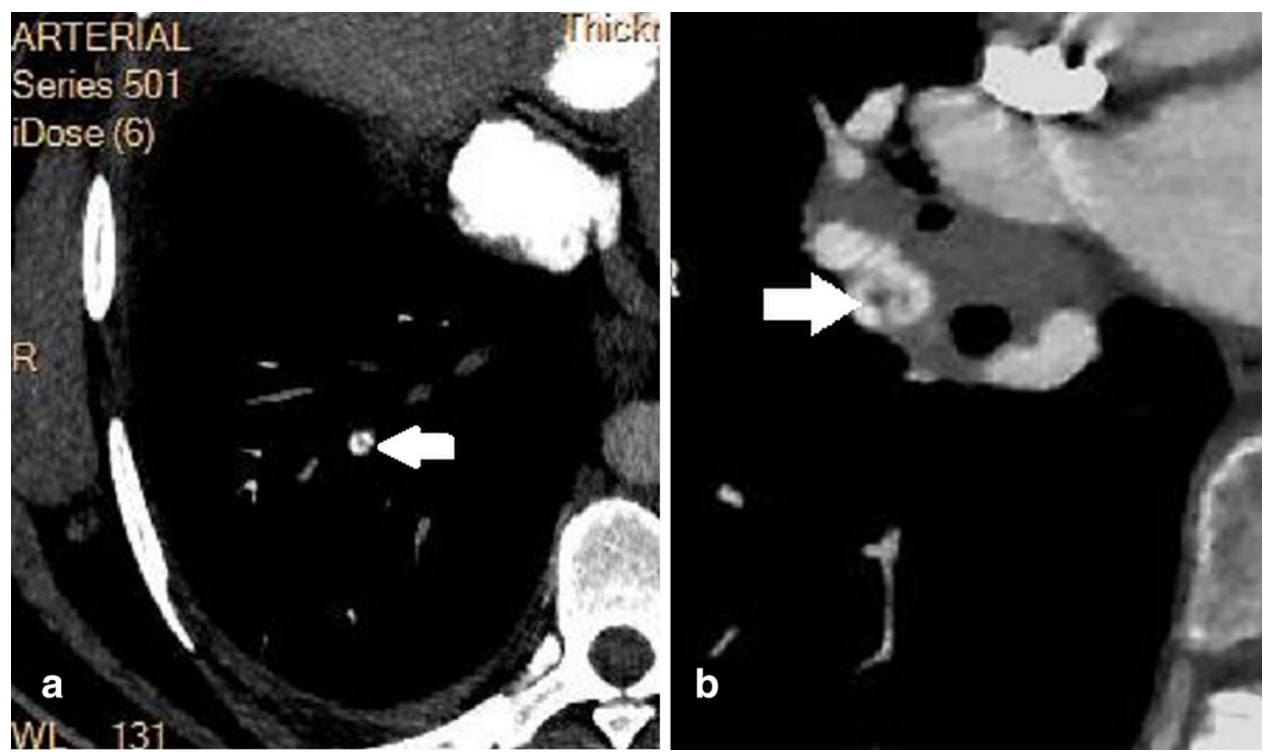

Fig. 11 CT pulmonary angiography in a patient of chronic pulmonary embolism showing a linear filling defect attached to the walls of a segmental artery of lower lobe of right lung (arrow) and (b) a web in the right lower lobar pulmonary artery (arrow) 


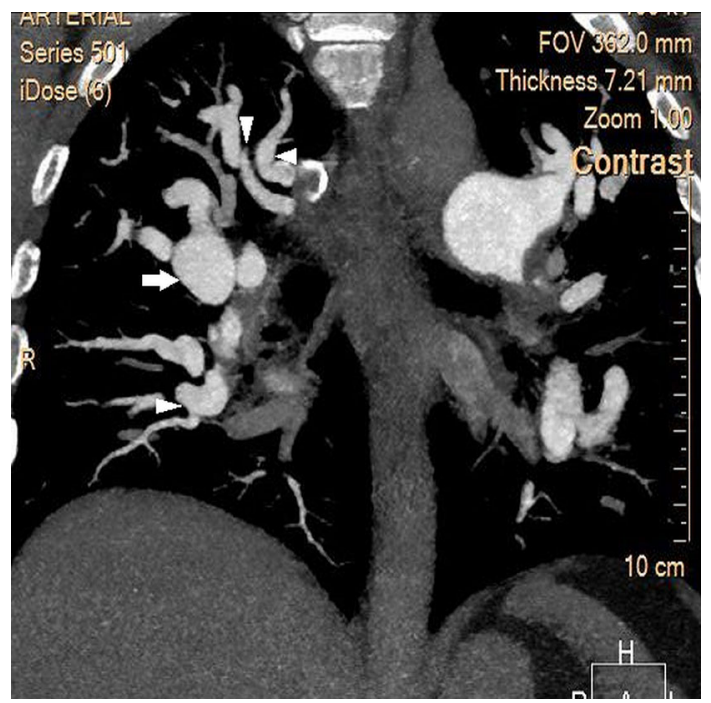

Fig. 13 CT pulmonary angiography image in a 45-year-old male patient of chronic pulmonary embolism showing tortuous arteries (arrowheads) and poststenotic aneurysmal dilatation in the segmental artery of right lung (arrow)

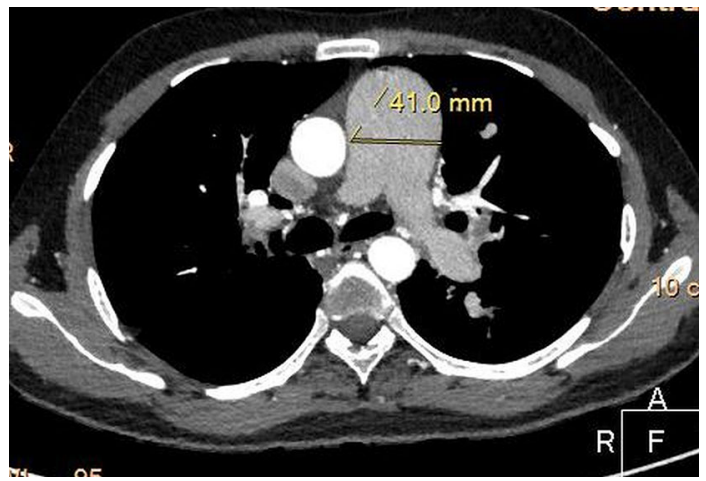

Fig. 14 A 55-year-old male patient of chronic pulmonary embolism. $\mathrm{CT}$ image shows dilatation of main pulmonary artery (solid line)

\section{Enlargement of Main Pulmonary Artery}

Central pulmonary arteries may be enlarged in chronic thromboembolism due to increased vascular resistance. Main pulmonary artery is considered enlarged, when its diameter is more than $29 \mathrm{~mm}$ or more than the diameter of the aorta (Fig. 14).

\section{Collateral Systemic Vessels}

Bronchial arteries are commonly enlarged in chronic thromboembolism due to decreased pulmonary artery blood flow (Fig. 15). The other systemic arteries which show enlargement in chronic thromboembolism are the intercostal, inferior phrenic, and internal mammary arteries [33].

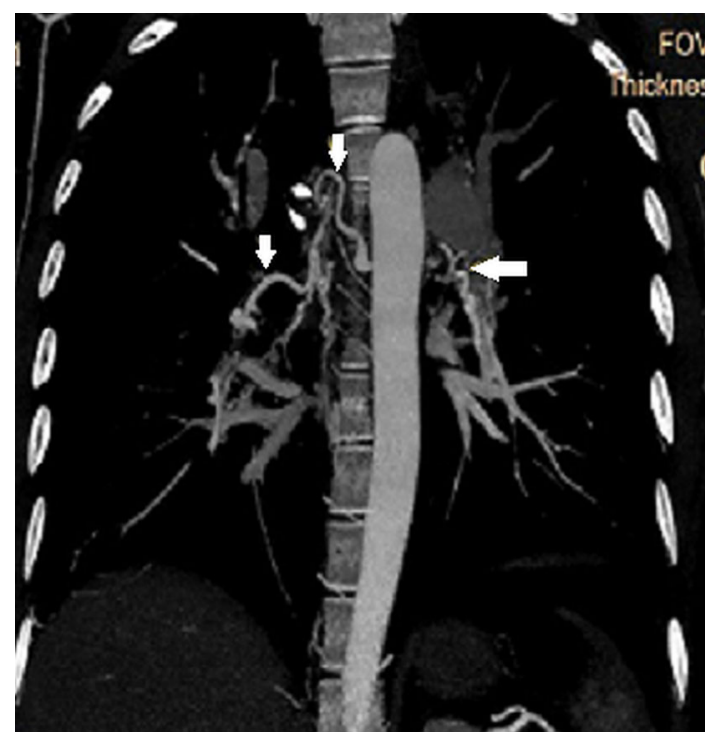

Fig. 15 CT pulmonary angiography image of a 60-year-old male with chronic pulmonary thromboembolism showing hypertrophic tortuous bronchial arteries (arrows)

\section{Lung Parenchymal CT Findings}

Mosaic pattern of attenuation of lung parenchyma is a common finding on MDCT in chronic thromboembolism (Fig. 16). It is a combination of decreased and increased attenuation areas in lung parenchyma due to nonuniform arterial perfusion. Decreased attenuation areas are due to low perfusion of blood flow distal to the obstruction, and increased attenuation areas are due to the redistribution of blood flow. In addition, wedge-shaped pleural-based opacities, irregular peripheral linear opacities (Fig. 16), peripheral nodules, and cavities may be seen in chronic thromboembolism on CT [34].

\section{Assessment of Clot Resolution and Severity of Pulmonary Embolism}

Pulmonary CT angiography may be used to quantitatively assess the rate of resolution of acute PE. Aghayev et al. [35•] in a retrospective study of 111 patients reported that during a follow up period of 1 year, 85 patients $(77 \%)$ showed complete resolution of $\mathrm{PE}$, and the rate of resolution was faster in peripheral pulmonary arteries as compared to the central pulmonary arteries. In addition to the diagnosis, pulmonary CT angiography can characterize and measure the clot burden which has been considered as a predictor of recurrent $\mathrm{PE}$, and clot burden measured can help to guide the duration of anticoagulation treatment [36]. Clot burden can be measured semiquantitatively or quantitatively using various scoring methods from pulmonary CT angiography images. Qanadli et al. [37] and Mastora et al. [38] proposed semiquantitative scoring systems, while Nakada et al. [39] 

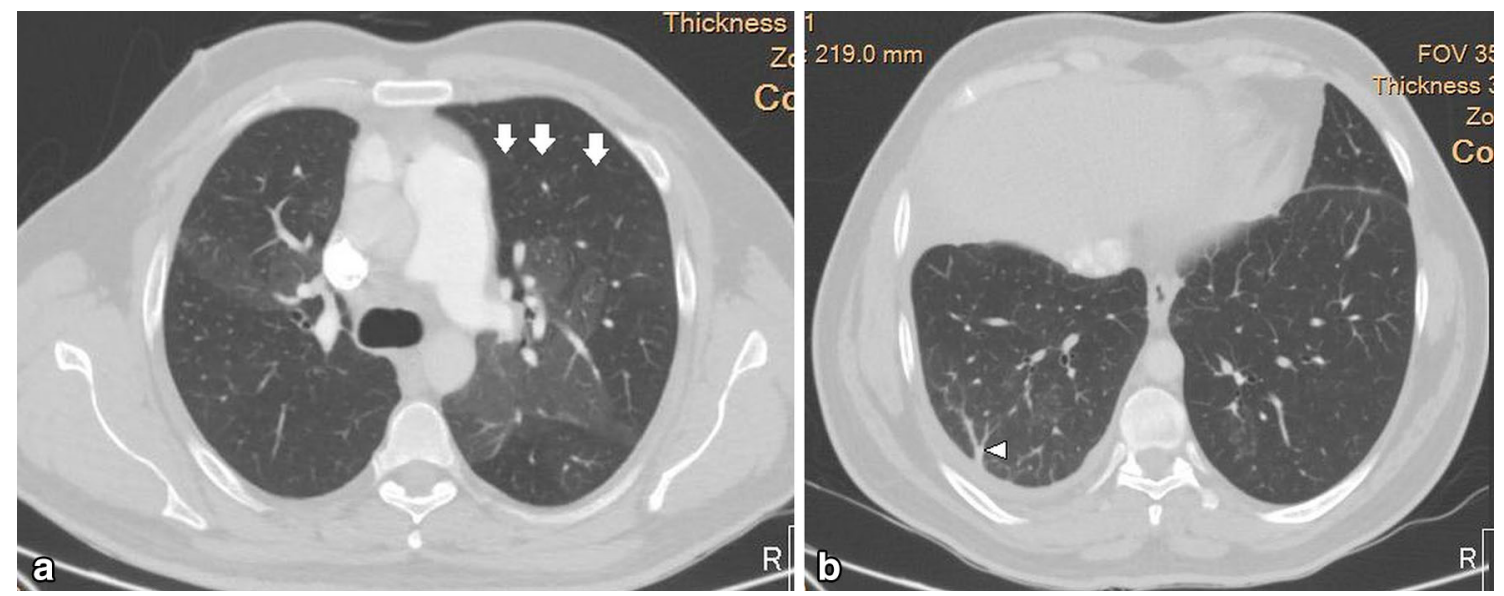

Fig. 16 A 60-year-old female patient of chronic pulmonary embolism. a CT image demonstrates mosaic perfusion pattern. Lowattenuation regions of under perfused lung show vessels (arrow) that

are smaller in size than the adjacent normally perfused lung. b CT scan of same patient showing irregular peripheral linear opacity (arrowhead)
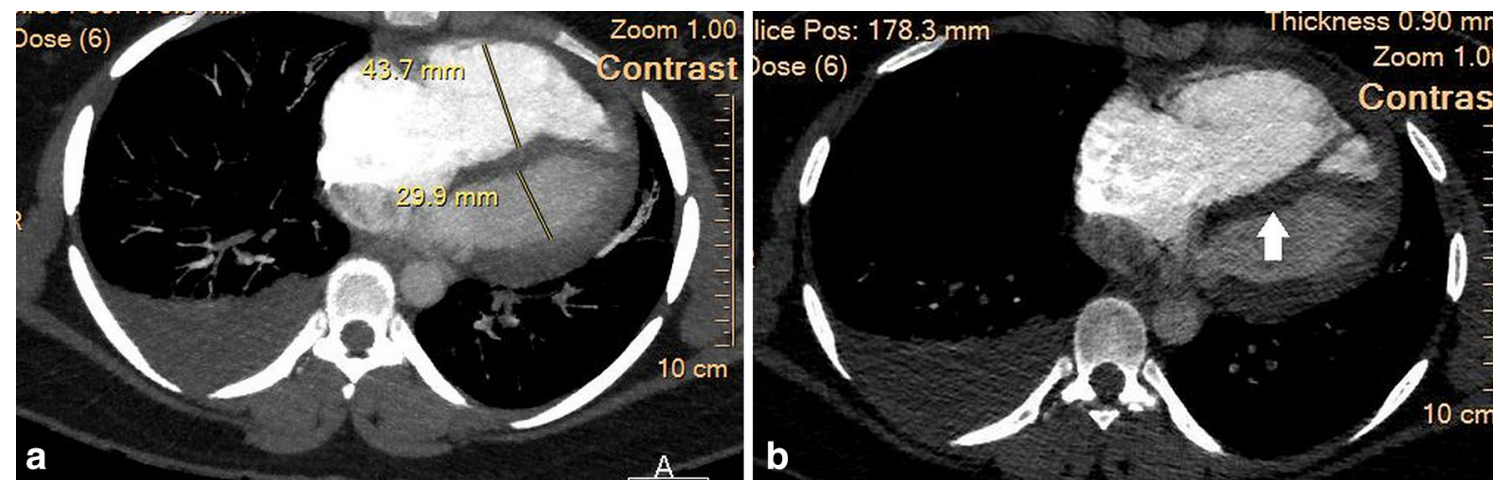

Fig. 17 A 50-year-old male patient of acute pulmonary embolism. a CT image shows short axis diameter of right ventricle more than the short axis diameter of the left ventricle $(\mathrm{RV} / \mathrm{LV}>1)$. b CT image shows leftward bowing of the interventricular septum (arrow)

and Furlan et al. [40] proposed quantitative systems to measure the clot burden that would help to assess the severity of PE.

Clot burden in PE has also been associated with the right heart dysfunction and mortality. Baptista et al. did an analysis of various radiological indices of right heart dysfunction, the ratio of right ventricle diameter to left ventricle diameter (RV/LV ratio), arterial obstruction index, the ratio of main pulmonary artery diameter to ascending aorta diameter (PA/AO ratio), and azygos vein diameter obtained from CT pulmonary angiography images to assess the medium-term prognostic impact in intermediate to high-risk PE patients. He observed that medium-term mortality due to PE was 11-fold higher in patients who had significantly high $\mathrm{RV} / \mathrm{LV}$ ratio [41•]. Kang et al. [42] reported that RV dysfunction parameters, especially $\mathrm{RV} / \mathrm{LV}$ ratio obtained from CT pulmonary angiography images, may predict the prognosis in acute PE. Furlan et al. in a retrospective study of 635 patients of $\mathrm{PE}$ found that
$\mathrm{RV} / \mathrm{LV}$ ratio and morphology of interventricular septum have the highest correlation with the clot burden. The study showed that the patients with right heart dilatation (RV/LV ratio) and flattened or bowing of interventricular septum had significantly larger clot burden (Fig. 17). This study also demonstrated that among the CT signs of right heart dysfunction, the RV/LV ratio, flattening or bowing of interventricular septum, PA/AO ratio, and reflux of contrast medium into the inferior vena cava (IVC), only RV/LV ratio, was associated with short-term mortality [43•].

\section{Conclusion}

MDCT pulmonary angiography has become the mainstay of the diagnosis of PE because of its high accuracy, rapid scanning, and widespread availability. MDCT is playing a crucial role not only in the diagnosis of PE, but also in assessing the severity and prognosis of PE. With the 
advances in the technology of MDCT, the sensitivity and specificity of CT pulmonary angiography will further improve with the help of newer techniques, and MDCT will play a pivotal role in the patients with suspected PE.

\section{Compliance with Ethics Guidelines}

Conflict of Interest Dr. Kishan Singh Rawat, Dr. Hariprasad Sudarsan, Dr. Anurag Yadav, and Dr. Samarjit Singh Ghuman each declare no potential conflicts of interest. Dr. T. B. S. Buxi is a section editor for Current Radiology Reports.

Human and Animal Rights and Informed Consent This article does not contain any studies with human or animal subjects performed by any of the authors.

\section{References}

Papers of particular interest, published recently, have been highlighted as:

- Of importance

1. Horlander KT, Mannino DM, Leeper KV. Pulmonary embolism mortality in the United States, 1979-1998: an analysis using multiple-cause mortality data. Arch Intern Med. 2003;163: 1711-7.

2. Giuntini C, Ricco GD, Marini C, et al. Pulmonary embolism: epidemiology. Chest. 1995; 107(suppl):3S-9S.

3. Lindblad B, Sternby NH, Bergqvist D. Incidence of venous thromboembolism verified by necropsy over 30 years. BMJ. 1991;302:709-11.

4. Alikhan R, Peters F, Wilmott R, Cohen AT. Fatal pulmonary embolism in hospitalised patients: a necropsy review. J Clin Pathol. 2004;57:1254-7.

5. Anderson FA Jr, Zayaruzny M, Heit JA, Fidan D, Cohen AT. Estimated annual numbers of US acute-care hospital patients at risk for venous thromboembolism. Am J Hematol. 2007;82: 777-82.

6. Wittram C, Maher MM, Yoo AJ, et al. CT angiography of pulmonary embolism: diagnostic criteria and causes of misdiagnosis. Radiographics. 2004;24:1219-38.

7. Stein PD, Kayali F, Olson RE. Trends in the use of diagnostic imaging in patients hospitalized with acute pulmonary embolism. Am J Cardiol. 2004;93:1316-7.

8. Prologo JD, Gilkeson RC, Diaz M, et al. CT pulmonary angiography: a comparative analysis of the utilization patterns in emergency department and hospitalized patients between 1998 and 2003. AJR Am J Roentgenol. 2004;183(4):1093-6.

9. Remy-Jardin M, Remy J, Wattinne L, Giraud F. Central pulmonary thromboembolism: diagnosis with spiral volumetric CT with the single-breathhold technique-comparison with pulmonary angiography. Radiology. 1992;185:381-7.

10. Goodman LR, Curtin JJ, Mewissen MW, et al. Detection of pulmonary embolism in patients with unresolved clinical and scintigraphic diagnosis:helical CT versus angiography. AJR Am J Roentgenol. 1995;164:1369-74.

11. Ghaye B, Szapiro D, Mastora I, et al. Peripheral pulmonary arteries: how far in the lung does multi-detector row spiral CT allow analysis? Radiology. 2001;219:629-36.
12. Schoepf U, Holzknecht N, Helmberger T, et al. Subsegmental pulmonary emboli: improved detection with thin-collimation multi-detector row spiral CT. Radiology. 2002;222:483-90.

13. Stein PD, Fowler SE, Goodman LR, PIOPEDII Investigators, et al. Multidetector computed tomography for acute pulmonary embolism. N Engl J Med. 2006;354:2317-27.

14. - Wittenberg R, Berger FH, Peters JF, et al. Acute pulmonary embolism: effect of a computer-assisted detection prototype on diagnosis-an observer study. Radiology. 2012; 262:305-13. CAD can play a role in the diagnosis of pulmonary embolism. This article emphasized that advances in technology may improve the detection of segmental and subsegmental pulmonary emboli.

15. Simon M, Boiselle PM, Choi JR, et al. Paddlewheel CT display of pulmonary arteries and other lung structures: a new imaging approach. AJR Am J Roentgenol. 2001;177(1):195-8.

16. Chiang EE, Boiselle PM, Raptopoulos V, et al. Detection of pulmonary embolism: comparison of paddlewheel and coronal CT reformations - initial experience. Radiology. 2003;228(2): 577-82.

17. van Rossum AB, Treuiriet FEE, Kieft GJ, Smith SJ, SchepersBok R. Role of spiral volumetric computed tomographic scanning in the assessment of patients with clinical suspicion of pulmonary embolism and an abnormal ventilation/perfusion lung scan. Thorax. 1996;51:23-8.

18. Hampton AO, Castleman B. Correlation of post-mortem chest teleroentgenograms with autopsy findings with special reference to pulmonary embolism and infarction. Am J Roentgenol Radium Ther. 1940;43:305-26.

19. Westermark N. On the roentgen diagnosis of lung embolism. Acta Radiol. 1938;19:357-72.

20. Sagel SS, Greenspan RH. Nonuniform pulmonary arterial perfusion: pulmonary embolism? Radiology. 1970;99:541-8.

21. Bachynski JE. Absence of the air bronchogram sign: a reliable finding in pulmonary embolism with infarction or hemorrhage. Radiology. 1971;100:547-52.

22. Woesner ME, Sanders I, White GW. The melting sign in resolving transient pulmonary infarction. Am J Roentgenol Radium Ther Nucl Med. 1971;111:782-90.

23. Grieco MH, Ryan SF. Aseptic cavitary pulmonary infarction. Am J Med. 1968;45:811-6.

24. Revel MP, Triki R, Chatellier G, et al. Is it possible to recognize pulmonary infarction on multisection CT images? Radiology. 2007;244:875-82.

25. Schwickert HC, Schweden F, Schild HH, et al. Pulmonary arteries and lung parenchyma in chronic pulmonary embolism: preoperative and postoperative CT findings. Radiology. 1994;191: $351-7$.

26. Auger WR, Kim NH, Trow TK. Chronic thromboembolic pulmonary hypertension. Clin Chest Med. 2010;31:741-58.

27. Castaner E, Gallardo X, Ballesteros E, et al. CT diagnosis of chronic pulmonary thromboembolism. Radiographics. 2009;29: $31-53$.

28. Wittram C, Kalra MK, Maher MM, Greenfield A, McLoud TC, Shepard JA. Acute and chronic pulmonary emboli: angiographyCT correlation. AJR Am J Roentgenol. 2006;186(6 suppl 2):S421-9.

29. Bergin CJ, Sirlin CB, Hauschildt JP, et al. Chronic thromboembolism: diagnosis with helical CT and MR imaging with angiographic and surgical correlation. Radiology. 1997;204:695-702.

30. Korn D, Gore I, Blenke A, Collins DP. Pulmonary arterial bands and webs: an unrecognized manifestation of organized pulmonary emboli. Am J Pathol. 1962;40:129-51.

31. Tardivon AA, Musset D, Maitre S, et al. Role of CT in chronic pulmonary embolism: comparison with pulmonary angiography. J Comput Assist Tomogr. 1993;17:345-51. 
32. Auger WR, Fedullo PF, Moser KM, Buchbinder M, Peterson KL. Chronic major-vessel thromboembolic pulmonary artery obstruction: appearance at angiography. Radiology. 1992;182:393-8.

33. Remy-Jardin M, Duhamel A, Deken V, Bouaziz N, Dumont P, Remy J. Systemic collateral supply in patients with chronic thromboembolic and primary pulmonary hypertension: assessment with multidetector row helical CT angiography. Radiology. 2005;235:274-81.

34. King MA, Ysrael M, Bergin CJ. Chronic thromboembolic pulmonary hypertension: CT findings. AJR Am J Roentgenol. 1998; 170:955-60.

35. - Aghayev A, Furlan A, Patil A,et al. The rate of resolution of clot burden measured by pulmonary CT angiography in patients with acute pulmonary embolism. AJR Am J Roentgenol. 2013; 200:791-7. This article described that peripheral segmental and subsegmental pulmonary emboli resolve faster than the central pulmonary emboli in contrast to the previous studies.

36. Agnelli G, Becattini C. Treatment of DVT: how long is enough and how do you predict recurrence. J Thromb Thrombolysis. 2008;25:37-44.

37. Qanadli SD, El Hajjam M, Vieillard-Baron A, et al. New CT index to quantify arterial obstruction in pulmonary embolism: comparison with angiographic index and echocardiography. AJR Am J Roentgenol. 2001;176:1415-20.

38. Mastora I, Remy-Jardin M, Masson P, et al. Severity of acute pulmonary embolism: evaluation of a new spiral CT angiographic score in correlation with echocardiographic data. Eur Radiol. 2003;13:29-35.

39. Nakada K, Okada T, Osada H, Honda N. Relation between pulmonary embolus volume quantified by multidetector computed tomography and clinical status and outcome for patients with acute pulmonary embolism. Jpn J Radiol. 2010;28:34-42.

40. Furlan A, Patil A, Park B, Chang CC, Roberts MS, Bae KT. Accuracy and reproducibility of blood clot burden quantification with pulmonary CT angiography. AJR Am J Roentgenol. 2011; 196:516-23.

41. - Baptista R, Santiago I, Jorge E, et al. One-shot diagnostic and prognostic assessment in intermediate to high-risk acute pulmonary embolism patients: the role of multidetector computed tomography. Rev Port Cardiol. 2013;32. This article described that $\mathrm{RV} / \mathrm{LV}$ ratio has a significant impact on medium-term mortality due to acute PE.

42. Kang DK, Thilo C, Schoepf UJ, et al. CT signs of right ventricular dysfunction: prognostic role in acute pulmonary embolism. JACC Cardiovasc Imaging. 2011;4:841-9.

43. - Furlan A, Aghayev A, Chang $\mathrm{CCH}$, et al. Short-term mortality in acute pulmonary embolism: clot burden and signs of right heart dysfunction at CT pulmonary angiography. Radiology. 2013; 265:283-93. This is one of the few articles which proved association between RV/LV ratio and mortality, and correlation between clot burden and right heart dilatation in acute PE. 